\title{
Disease Management in Patients with Delirium
}

\author{
Thomas Jochum ${ }^{1}$ and Karl-Jürgen Bär ${ }^{*, 2}$ \\ ${ }^{I}$ Department of Psychiatry and Psychotherapy, Straße des Friedens 122, SRH Waldklinikum, Gera 07548, Germany \\ ${ }^{2}$ Department of Psychiatry and Psychotherapy, Philosophenweg 3, University Hospital, Jena 07749, Germany
}

\begin{abstract}
Delirium occurs as a pathophysiological state in connection with various diseases, particularly among patients in critical care. Since it may be a life-threatening condition, any physician should be able to initiate correct assessment and treatment of delirium. This review summarizes current knowledge on care of patients with delirium.

The symptoms of delirium contain primarily disturbance of consciousness, attention, cognition, and perception and may comprise disturbance of psychomotor activity, emotions, and sleep. High prevalence of delirium is observed in critically ill patients in intensive care units. It is thus necessary to monitor the comorbid conditions when patients are diagnosed with delirium, especially since delirium is a prognostic factor for rise in morbidity and mortality. The identification of underlying aetiology of delirium and immediate intervention and treatment for urgent general medical conditions should be in the focus, and should be paralleled by measures ensuring patient's safety and continuous monitoring.

Somatic interventions depend on underlying aetiology, and patient's clinical as well as comorbid conditions, but they mainly consist of treatment with high-potency neuroleptic drugs such as haloperidol. Interventions which reduce or eliminate environmental factors contributing to an exacerbation and maintenance of delirium are strongly recommended.

Eventually, it is important to establish and maintain psychological support to the patient and his family regarding illness, including post-delirium management.
\end{abstract}

Keywords: Delirium, treatment, psychiatric, assessment, aetiology.

\section{CLINICAL FEATURES}

Delirium is defined as an acute, severe and principally reversible organic brain syndrome of different aetiology. Clinical features of delirium include disturbances of consciousness, attention, cognition, perception, thinking, emotions, wake-sleep rhythm, and psychomotor activity. Symptoms develop over hours to days and they tend to fluctuate during the course of the disease. Some patients suffer from prodromal symptoms such as restlessness, anxiety, irritability or sleep disturbance 1 to 3 days before the onset of full-blown delirium. While symptoms typically last for 10 to 12 days, durations from less than 1 week to more than 2 months is possible [1-7].

\section{Definition}

According to the International classification of diseases, diagnosis of delirium can be made when the following criteria are fulfilled [8]:

1. Disturbance of attention and consciousness with reduced ability to focus, sustain, or shift attention up to stupor or coma.

2. Global disturbance of cognition (illusions, hallucinations, most often visual), perception, delusional ideas, alteration of especially recent memory and abstract thinking, incoherence, disorientation (most often to time or place), language and speech disturbances (dysarthria, visuo-constructional impairment).

*Address correspondence to this author at the Department of Psychiatry and Psychotherapy, University Jena, Philosophenweg 3, Jena 07740, Germany; Tel: +49-3641-935282; Fax: +49-3641-936217;

E-mail: Karl-Juergen.Baer@med.uni-jena.de
3. Disturbance of psychomotor activity (hypo - or hyperactivity, prolonged reaction time, increase startle response, logorrhoea and mutism).

4. Disturbance of wake-sleep rhythm (e.g. inversion of wake-sleep-rhythm, nightmares, daytime sleepiness, night-time agitation, sleep discontinuity).

5. Affective symptoms like anxiety, fear, depression, irritability, euphoria, anger and apathy. Patients may also show emotional lability.

If those symptoms cannot be better explained by preexisting dementia and represent sudden decline from previous functioning level, the diagnosis of delirium can be made. The diagnosis is substantiated if evidence exists from the patient's history, physical examination, or from laboratory tests. Most often the delirium is a consequence of severe general medical condition, substance intoxication or withdrawal, use of medication, toxin exposure or combination of these factors.

\section{Epidemiology}

Prevalence of delirium in hospitalised medically ill ranges from 10 to $30 \%$ in younger patients [9] and reaches up to $65 \%$ in the hospitalised elderly [10]. About $50 \%$ of postoperative patients (especially patients who just underwent cardiotomy, hip surgery, or transplant) [11, 12], $28-48 \%$ of patients with advanced cancer upon admission to acute hospital or hospice [13], and $85 \%$ up to $90 \%$ of patients in days and hours before death develop delirium [14, 15]. There is also an increased risk to develop delirium for patients with burns and patients who receive dialysis $[16,17]$ 
or suffering from chronic liver diseases [18]. Furthermore, approximately $30-40 \%$ of hospitalized AIDS patients develop delirium [19-21]. In postoperative patients (especially after orthopaedic surgery), delirium is an indication for postoperative complications, longer postoperative recuperation periods, longer hospital stays, and long-term disability [22-24].

Delirium can appear at every age, but it is most often observed in patients older than 60 years [3]. Delirium can occur in parallel with dementia and is one of the important differential diagnoses besides affective disorder in the elderly patient $[25,26]$. The most common issue in differential diagnosis here is to determine whether patient has dementia rather than delirium. It is also possible that delirium is superimposed on pre-existing dementia. The temporal course and reversibility of symptoms of cognitive deficiency are helpful in distinguishing between delirium and dementia. Patients with delirium typically show fluctuating symptoms over a time period of $24 \mathrm{~h}$, including consciousness, whereas these symptoms usually appear unaltered in patients with dementia [25].

\section{Clinical Picture}

From the practical point of view, it seems to be of interest that some authors differentiate delirium in two different subtypes according to psychomotor activity. These delirium subtypes include hyperactive (agitated, hyper alert) subtype, the hypoactive (lethargic, hypo alert) and mixed subtype [27]. The hyperactive form is thought to occur with hallucinations, delusions or illusions, whereas the hypoactive form is characterised by confusion and sedation. The delirious patient may also exhibit neurological abnormalities (e.g. tremor, myoclonus, nystagmus, ataxia, asterixis, cranial nerve palsies and reflex or muscle tone changes) depending on aetiology [18]. EEG background rhythm is typically showing generalised slowing, except in alcohol or sedative withdrawal syndrome with delirium, where EEG shows fast activity [28]. Several screening instruments have been designed to screen for delirium symptoms among patients by nursing stuff (e.g., Clinical Assessment of Confusion-A, Confusion Rating Scale, Confusion Assessment Method, see appendix 1) [18].

\section{Long-Term Outcome}

In general, delirium in the medically ill and elderly patients is associated with significant morbidity and mortality $[29,30]$. Patients with delirium have $25 \%$ chance to die within the following 6 months [31]. In the presence of delirium on admission to the hospital the mortality rate was found to be $22 \%-76 \%$ in elderly patients [29, 32]. Delirium, particularly if untreated, may progress to stupor, coma, seizures, or death. Seizures can occur as a complication among patients with delirium due to alcohol or sedative withdrawal, cocaine intoxication, hypoglycaemia, strokes, head trauma, or extensive burns [18].

Most patients who suffer from delirium recover within 4 weeks or shorter period of time, but a fluctuating course up to 6 months is possible. An important risk factor for the prolonged course of delirium is age, as elderly patients frequently show symptom durations exceeding 1 month [18, 33]. Full recovery is also less likely in the elderly, although symptoms may be due to pre-existing dementia. Additionally, elderly patients have significantly increased risk of developing complications, such as pneumonia and decubitus ulcers [34].

\section{UNDERLYING AETIOLOGIES}

Because delirium is potentially life threatening, it is absolutely necessary to perform careful and comprehensive assessment of general medical and mental state of the patient. Although clinical presentation of delirium is quite common, the underlying aetiologies differ. When assessing the potential diagnosis of delirium, clear organic aetiology is the most probable cause [5] but several environmental factors appear to increase the severity of symptoms of delirium [35]. Since about $44 \%$ of the critically ill and elderly hospitalised patients show multiple aetiologies for delirium [36], careful clinical observation is the most important measure for the treating clinicians [18]. Aetiologies that can be causes for delirium are detailed below (see table 1).

\section{Delirium due to General Medical Condition}

Delirium can be associated with many different general medical conditions. Some of them have typical clinical and paraclinical findings, which can be helpful for diagnosis of underlying medical disturbance. In general, underlying condition can be central nervous system disorders, metabolic disorders, cardiopulmonary disorders and systemic illnesses.

\section{Delirium due to Substance Use or Withdrawal}

Substances that are not usually regarded as having psychoactive properties as well as those with established psychoactive properties are able to cause delirium due to substance use or withdrawal [37]. Delirium that occurs during substance intoxication may arise within minutes to hours. Potential for causing delirium is much higher when drugs having anticholinergic activity are combined [38]. With decreasing intoxication, delirium generally ends within hours or a few days after intoxication [18]. In the case of substance withdrawal, delirium develops after reduction of sustained high dose use or when clearance of a certain substance is improved. Substance withdrawal delirium may last only a few hours or may persist for as long as 2-4 weeks. Longer acting substances are usually associated with less severe but more protracted delirium [18]. The following substances (including substances of abuse, prescription medication, toxins) are associated with delirium:

1) Drugs of abuse: Alcohol, amphetamines, cannabis, cocaine, opiods, hypnotics, sedatives, inhalants, hallucinogens, phencyclidine,

2) Medications: Anaesthetics, analgesics, antiasthmatic drugs, antihistamines, anticonvulsants, antihypertensive and cardiovascular medications, antimicrobials, antiparkinsonian medications, corticosteroids, gastrointestinal medication, muscle relaxants, immunosuppressive agents, lithium and anticholinergic psychotropic medications,

3) Toxins: Anticholinesterase, organophosphate insecticides, carbon monoxide and dioxide, volatile substances such as fuel and organic solvents. 
Table 1. Possible General Conditions that Can Cause Delirium

\begin{tabular}{|c|c|}
\hline Central nervous system disorders & $\begin{array}{l}\text { vascular disease (ischemic stroke, subdural haematoma, subarachnoid haemorrhage, intracranial bleeding) } \\
\text { hypertensive encephalopathy } \\
\text { head trauma } \\
\text { seizure (post-ictal state, non-convulsive status epilepticus) } \\
\text { meningitis/meningo-encephalitis (bacterial, viral, septic metastatic, abscess of the brain, HIV, mycosis etc.) } \\
\text { septic encephalopathy } \\
\text { degenerative disease }\end{array}$ \\
\hline Metabolic disorders & $\begin{array}{c}\text { renal failure } \\
\text { hepatic failure } \\
\text { anaemia } \\
\text { hypoxia } \\
\text { hypoglycaemia } \\
\text { thiamine deficiency } \\
\text { endocrinopathy } \\
\text { fluid imbalance } \\
\text { electrolyte imbalance } \\
\text { acid-base imbalance }\end{array}$ \\
\hline Cardio-pulmonary disorders & $\begin{array}{l}\text { myocardial infarction } \\
\text { congestive heart failure } \\
\text { cardiac arrhythmia } \\
\text { shock } \\
\text { respiratory failure }\end{array}$ \\
\hline systemic illnesses & $\begin{array}{l}\text { Substance intoxication } \\
\text { Substance withdrawal } \\
\text { postoperative state } \\
\text { infection } \\
\text { neoplasm } \\
\text { severe trauma } \\
\text { temperature dysregulation }\end{array}$ \\
\hline
\end{tabular}

\section{TREATMENT RATIONALES}

\section{Assessment and General Aspects of Treatment}

Treatment of delirium involves combination of pharmacological, environmental and supportive interventions. An appropriate treatment must combine interventions in order to identify and correct the underlying causes with interventions that relieve current symptoms. The physician caring for the patient should coordinate with other specialists (internist, neurologist, surgeon, psychiatrist etc.) to ensure a comprehensive assessment and treatment. The goal of diagnosis is to discover reversible causes of delirium and prevent complications by prompt treatment of these specific disorders [18, 26].

First steps in identifying aetiology are a careful review of patient's medical history and medication, including general medical or anaesthesia records (if postoperative), physical, neurological and psychiatric examination, and an interview of family members or other persons close to the patient. Blood chemistries (electrolytes, glucose, calcium, albumin, blood urea nitrogen, creatinine, SGOT, SGPT, bilirubin, alkaline phospatase, magnesium, $\mathrm{PO}_{4}$ ), complete blood count, electrocardiogram, chest-X-ray, arterial blood gases, and urine analysis are indicated for all patients suffering from delirium.
Additional investigations should be organized as stag-tostage diagnostic measures if they are clinically indicated and practicable: blood and urine culture, drug screen and serum levels of medications (e.g. theophylline, cyclosporine, digitoxine) as well as special blood tests (ammonia, venereal disease research laboratory, folate, $\mathrm{B}_{1}, \mathrm{~B}_{6}$ and $\mathrm{B}_{12}$ levels, heavy metal screen, lupus erythematosus prep, antinuclear antibodies, urinary porphyrins, HIV) should be done following clinical suspicion. If cerebral trauma, tumour, metastases, neurovascular or infectious diseases are thought to be possible causes of delirium, computerized brain tomography $(\mathrm{CT})$ or magnetic resonance imaging (MRI) must be done. If central nervous infection is considered lumbar puncture and analysis of cerebrospinal fluid are mandatory. Finally, electroencephalogram (EEG) could clarify if an epileptic seizure (especially non-convulsive status epilepticus) is responsible for the delirious symptomatology. Neuro-imaging, lumbar puncture and EEG also should be done always if the cause of delirium remains unclear, especially in younger patients.

\section{Immediate Interventions}

Interventions for acute conditions should be initiated in parallel to the diagnostic process. Vital signs, fluid intake and output, and levels of oxygenation must be continuously monitored as delirium can be a sign for life-threatening general medical condition [18]. Generally, nonessential 
medications should be discontinued, and doses of necessary medication should be kept as low as possible. The following measures should be taken depending on symptoms and aetiology:

- In hypoxia or anoxia, immediate oxygen delivery is indicated. This can be the case in context of pneumonia, obstructive or restrictive pulmonary or cardiac disease, hypotension, severe anaemia, or carbon monoxide poisoning.

- Diagnosis of hypoglycaemia can be confirmed by blood and urine tests. It can be easily treated with 50 $\mathrm{mg} \mathrm{50 \%} \mathrm{glucose} \mathrm{and} 100 \mathrm{mg}$ thiamine hydrochloride i.v. (before glucose).

- Hypertension of $250 / 150 \mathrm{mmHg}$ should lead to prompt antihypertensive treatment, for instance with clonidine.

- Alcohol or sedative withdrawal must be treated appropriately with substituting drugs and neuroleptics (diazepam plus haloperidol), reduced according to a schedule and complemented completed with thiamine hydrochloride initially i.v.

- Patients with Wernicke's encephalopathy require urgent treatment with $100 \mathrm{mg}$ i.v. thiamine hydrochloride followed by daily administration of the drug.

- In anticholinergic delirium, withdrawal of offending agents is indicated. In severe cases administration of physiostigmine should be considered unless contraindicated.

- In the context of psychopathology, suicidal tendencies and potential for violence must be assessed and if necessary interventions for risk minimization have to be taken (removal of dangerous items, increase in supervision, pharmacotherapy).

Assuring patient's safety also includes reducing risk for self-harm through falls, wandering etc. Patients who are restrained must be observed continuously, and restraints as well as their justification must be documented. Restraints themselves can increase agitation and carry risk for injuries. If possible, other protection such as sitters should be chosen. Regular monitoring will also allow for the adjustment of treatments, as it may indicate effectiveness of interventions [18].

\section{Somatic Interventions}

\section{Antipsychotics}

Numerous case reports and uncontrolled trials have provided evidence for efficacy of antipsychotics in treatment of delirium. Haloperidol, a high potency dopamine-blocking agent with few or no anticholinergic effects, minimal cardiovascular side effects, and no active metabolites, has generally been considered the antipsychotic medication of first choice for treatment of delirium [18, 39, 40]. Although haloperidol is the most studied pharmacological treatment, there has been an increasing use of risperidone in recent years [26, 41]. However, new antipsychotic medications such as risperidone, olanzapine, quetiapine etc. have not been studied intensively [42-44]. Studies have demonstrated superiority of antipsychotic medication over benzodiazepines in delirium treatment [45]. In patients treated with neuroleptics, improvement in delirium symptoms can be observed quickly and before interventions directed at medical aetiology of this delirium are initiated [40].

On the basis of doses used in several studies, starting haloperidol in range of 1-2 mg every 2-4 hours has been suggested for young adults and low doses $(0.25-0.50 \mathrm{mg}$ haloperidol every 4 hours) are recommended for elderly patients [18, 46, 47]. Titration to higher doses may be required for patients who continue to be agitated. Even bolus intravenous haloperidol doses exceeding $50 \mathrm{mg}$ with total daily dose up to $500 \mathrm{mg}$ have been reported and were associated with minimal effects on heart rate, blood pressure, and minimal extrapyramidal side effects $[18,48]$.

The most important side effects in the use of haloperidol are extrapyramidal effects, tardive dyskinesia and lengthening of QT interval. Development of torsades de pointes is associated with higher intravenous doses of haloperidol $(>35 \mathrm{mg}$ i.v.) and can be triggered by disturbances of serum electrolytes levels. Prolongation of $\mathrm{QT}_{\mathrm{c}}$ interval greater than $450 \mathrm{~ms}$ or to greater than $25 \%$ of that in previous ECGs may warrant telemetry, cardiology consultation, and dose reduction or discontinuation. Furthermore, serum level of magnesium and potassium should be monitored during haloperidol treatment [49-52].

Although there is no approval by clinical studies low potency antipsychotic drugs (melperone, pipamperone) might help to control symptoms like anxiety and restlessness in patients with delirium. Nevertheless these drugs should be used with caution as long they have anticholinergic side effects.

\section{Benzodiazepines}

Available data suggest that benzodiazepine monotherapy is ineffective as treatment for general cases of delirium [21, 45]. Nevertheless, benzodiazepines are the treatment of choice for delirium tremens or for delirium due to benzodiazepine withdrawal. Furthermore, benzodiazepines are an useful alternative to neuroleptics in clinical circumstances with need for anticonvulsive medication or anticholinergic side effects or akathisia caused by neuroleptics would seriously exacerbate a patient's condition [18]. The combination of antipsychotics and benzodiazepines decreases medication side effects and potentially increases clinical effectiveness, especially in severely ill cancer or AIDS patients [19, 21]. Adverse effects (sedation, behavioural disinhibition, amnesia, ataxia, respiratory depression, physical dependence, rebound insomnia, withdrawal reactions, and delirium) might occur, especially when using long acting benzodiazepines [53]. The risk for the development of these complications is greater in elderly patients [18]. Benzodiazepines are contraindicated in the treatment of delirium from hepatic encephalopathy, as well as in patients with respiratory insufficiency. When benzodiazepines are used, relatively short-acting medications with no active metabolites (e.g. lorazepam) should be selected, except in cases of delirium due to alcohol or sedative-hypnotic withdrawal. Doses must be carefully 
considered, given the possibility that benzodiazepines may exacerbate symptoms of delirium [45].

\section{Other Substances}

Physiostigmine, as a centrally cholinergic medication, has been used almost exclusively in cases of delirium clearly caused by anticholinergic medications [54]. Studies and case reports have shown that physiostigmine relieves delirium caused by atropine, scopolamine, ranitidine, homatropine eye drops, benztropine and meperidine. Possible side-effects are bradycardia, seizures, nausea, vomiting, salivation, and increased gastrointestinal acid and liver enzyme levels [42]. If necessary, physiostigmine should be administered parenterally in doses of 2-4 $\mathrm{mg}$ or as a continuous infusion of $4 \mathrm{mg} /$ hour $[18,55]$.

Deficiencies of vitamins such as nicotine amide, vitamin $\mathrm{B}$ and $\mathrm{C}$, thiamine and folic acid can also cause delirium. In general, any alcoholic or malnourished patient with delirium should be given multivitamin replacement [18].

Patients with agitated delirium and hypercatabolic conditions (e.g., hyperdynamic heart failure, adult respiratory distress syndrome, hyperthyroid storm) which are unresponsive to other pharmacologic interventions require paralytic agent as well as mechanical ventilation and should be heavily sedated [56]. Morphine or other opiates are an important palliative treatment in cases of delirium where pain is an aggravating factor, although some opiates can exacerbate delirium through anticholinergic activity of their metabolites [53] (for a conclusion see also appendix 2).

\section{CLINICAL FEATURES INFLUENCING TREAT- MENT}

\section{Co-Morbidity}

Several comorbid psychiatric disorders are influencing treatment of delirium. Since medications for psychiatric disorders, especially antidepressants and anxiolytic drugs, can cause and exacerbate delirium, treatment of other psychiatric disorders should only be started after recovery from delirium [18].

Furthermore, depression or dementia can only be diagnosed during delirium when patient's history reveals symptoms that clearly existed before onset of delirium, otherwise they might be misdiagnosed.

Some comorbid general medical conditions are also influencing treatment of delirium. First, patients at advanced age and especially nursing home patients have an increased risk for developing a delirium [26]. This can be caused by multiple general medical problems, polypharmacy, reduced hepatic metabolism, multisensory decline and other mental diseases, such as dementia. After conducting a careful medical evaluation, including assessment of the level of oxygenation and possible occult infections (e.g., urinary tract infections), the next step should be a screening of the patient's medication file for drugs with potential anticholinergic effects $[57,58]$. When initiating an antipsychotic medication, low doses should be used since elderly patients have a greater risk for extrapyramidal side effects and falls. However, if extrapyramidal side effects occur early in neuroleptic treatment of delirium and symptoms show fluctuations in course, Lewy body disease should be considered in differential diagnosis.

Another clinical feature influencing treatment is a hepatic disease. Impaired therapeutic effects and occurrence or increase of medication side effects can result from an altered transformation, reduced production of albumin in liver tissue and consequently raised plasma levels of medication. Some benzodiazepines, such as diazepam, require hepatic oxidation and can accumulate in patients with hepatic insufficiency. Other benzodiazepines (lorazepam, temazepam and oxazepam), and haloperidol only require glucuronidation as a major clearance pathway and should therefore be preferred in treatment of delirium in those patients [59].

Haloperidol should also be used in lower doses in AIDS patients, as those patients may have an increased sensitivity to extrapyramidal side effects [60].

\section{Disease Management}

Management of delirium includes the reduction of environmental factors that might trigger or worsen the disease or hinder recovery. Sleep wake cycle can be disturbed by intensive care unit equipment (pumps and respirators with alarms and beeps) and feeling of "timelessness" due to constant lighting can lead to overstimulation. Nonpharmacological sleep assistance (back massage, relaxation music, and warm drink at bedtime) and noise-reduction strategies (vibrating beepers, silent pillcrushers, quiet hallways, rescheduling procedures to allow sleep) help to reduce use of sedative drugs for sleep [61, 62]. It is important to provide regular amount of modest stimulation (vocal, visual, tactile). Understimulation leaves patient with his own internal disorganised perceptions and thoughts. The availability and functionality of patient's glasses or hearing aids play an important role in enabling sufficient stimulation. It also may be useful to recommend that family and friends spend time in patient's room and bring along familiar objects from home to provide orientation and feeling of security [61-63]. Early mobilization (active range-of-motions exercises three times daily, reduction of immobilizing equipment such as bladder catheters) and sufficient oral intake of fluids (including early cognition of dehydration, fluid intake protocol) reduce influence of risk factors immobility and dehydration [64]. Early and multicomponent intervention is probably most effective strategy. Once delirium has occurred, these arrangements are less effective [64].

\section{CONCLUSION}

As delirium might be life-threatening and has been shown as a prognostic factor for rise in morbidity and mortality it is necessary to identify underlying aetiology. Furthermore immediate intervention and treatment should be paralleled by measures ensuring patient's safety and continuous monitoring. Beside of somatic interventions treatment with high-potency neuroleptic drugs are strongly recommended. Establishing of psychological support to the patient and his family regarding illness, including postdelirium management must complete therapeutic actions. 


\section{APPENDIX 1}

Evaluator:

Date:

I. Acute onset and fluctuating course

a) Is there evidence of an acute change in mental status from the patient's baseline?

b) Did the (abnormal) behaviour fluctuate during the day, that is tend to come and go or increase and decrease in severity?

\section{Inattention}

Did the patient have difficulty focusing attention, for example, being easily distractible or having difficulty keeping track of what was being said?

\section{Disorganized thinking}

Was the patient's thinking disorganized or incoherent, such as rambling or irrelevant conversation, unclear or illogical flow of ideas, or unpredictable switching from subject to subject?

\section{Altered level of consciousness}

Overall, how would you rate the patient's level of consciousness?

-- Alert (normal)

-- Vigilant (hyperalert)

-- Lethargic (drowsy, easily aroused)

-- Stupor (difficult to arouse)

-- Coma (unarousable)

Do any checks appear in this box?

\section{Box 1}

\begin{tabular}{l|l} 
No & Yes \\
Yes \\
No \\
Yes
\end{tabular}

\section{Box 2}

No

Yes

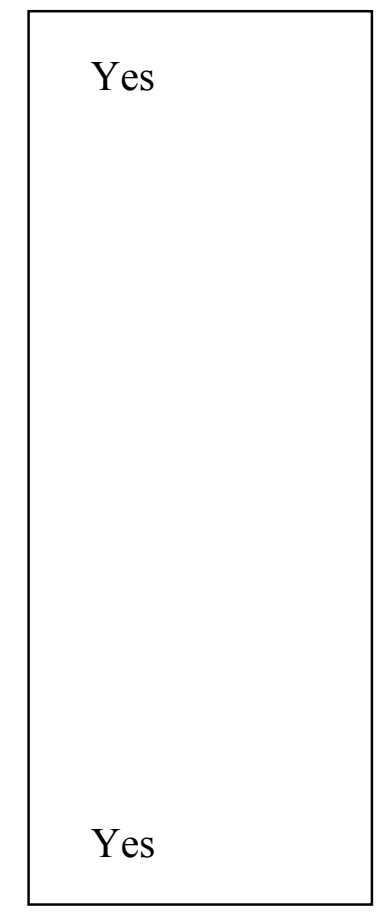

If all items in Box 1 are checked and at least one item in Box 2 is checked a diagnosis of delirium is suggested. 


\section{APPENDIX 2}

\section{Choice of Specific Somatic Interventions}

1. Antipsychotic medication

a) Haloperidol

- Initial dose 1-2 mg every 2-4 hours (adapted dose in elderly patients)

- Administered orally, intramuscularly, or intravenously

- Monitor ECG is required if given intravenously (QTc prolongation)

b) Newer antipsychotic drugs (risperidone, olanzapine, quetiapine)

- Only a few published clinical trials in delirium

\section{Other interventions}

a) Benzodiazepines

- In combination with haloperidol for alcohol/sedative-hypnotic withdrawal and in cases with prominent anxiety and agitation

- Combined treatment with haloperidol and 0.5-1.0 mg i.v. lorazepam

b) Cholinergics

- For delirium caused by anticholinergic agents

c) Opiods

- For agitation in palliative patients with delirium in whom pain is a aggravating factor

d) Paralysis and ventilation

- For agitated patients with agitated delirium and severe hypercatabolic conditions

\section{REFERENCES}

Manos PJ, Wu R. The duration of delirium in medical and postoperative patients referred for psychiatric consultation. Ann Clin Psychiatry 1997; 9(4): 219-26.

[2] Rockwood K. Acute confusion in elderly medical patients. J Am Geriatr Soc 1989; 37(2): 150-4.

[3] Rockwood K. The occurrence and duration of symptoms in elderly patients with delirium. J Gerontol 1993; 48(4): M162-6.

[4] Sirois F. Delirium: 100 cases. Can J Psychiatry 1988; 33(5): 375-8.

[5] Koponen H, Stenbäck U, Mattila E, Soininen H, Reinikainen K, Riekkinen P. Delirium among elderly persons admitted to a psychiatric hospital: clinical course during the acute stage and oneyear follow-up. Acta Psychiatr Scand 1989; 79(6): 579-85.

[6] Koizumi J, Shiraishi H, Ofuku K, Suzuki T. Duration of delirium shortened by the correction of electrolyte imbalance. Jpn J Psychiatry Neurol 1988; 42(1): 81-8.

[7] Andrew M, Freter S, Rockwood K. Prevalence and outcomes of delirium in community and non-acute care settings in people without dementia: a report from the Canadian Study of Health and Aging. BMC Med Genet 2006; 23: 1-5.

[8] World Health Organization: The ICD-10 classification of mental and behavioural disorders. Clinical Descriptions and Diagnostic Guidelines. 4th reviewed version ed. Geneva; 2002. 
[9] Fish D. Treatment of delirium in the critically ill patient. Clin Pharm 1991; 10(6): 456-66.

[10] Kalisvaart K, Vreeswijk R. Prevention of delirium in the elderly. Rev Esp Geriatr Gerontol 2008; 43: 19-24.

[11] Tan M, Felde A, Kuskowski M, et al. Incidence and predictors of post-cardiotomy delirium. Am J Geriatr Psychiatry 2008; 16(7).

[12] Santana SF, Wahlund L, Varli F, Tadeu VI, Eriksdotter Jonhagen M. Incidence, clinical features and subtypes of delirium in elderly patients treated for hip fractures. Dement Geriatr Cogn Disord 2005; 20(4): 231-7.

[13] Bruera E, Bush S, Willey J, et al. Impact of delirium and recall on the level of distress in patients with advanced cancer and their family caregivers. Cancer 2009; 115(9): 2004-12.

[14] Lawlor PG, Gagnon B, Mancini IL, et al. Occurrence, causes, and outcome of delirium in patients with advanced cancer: a prospective study. Arch Intern Med 2000; 27; 160(6): 786-94.

[15] Fainsinger RL, De Moissac D, Mancini I, Oneschuk D. Sedation for delirium and other symptoms in terminally ill patients in Edmonton. J Palliat Care 2000; 6(2):5-10.

[16] Antoon AY, Volpe JJ, Crawford JD. Burn encephalopathy in children. Pediatrics 1972; 50(4): 609-16.

[17] Burn DJ, Bates D. Neurology and the kidney. J Neurol Neurosurg Psychiatry 1998; 65(6): 810-21.

[18] APA. Practice guidline for the treatment of patients with delirium. American Psychiatric Association Practice Guidlines 1999; first ed.

[19] Fernandez F, Levy JK. Psychopharmacotherapy of psychiatric syndromes in asymptomatic and symptomatic HIV infection. Psychiatr Med 1991; 9(3): 377-94.

[20] Perry SW. Organic mental disorders caused by HIV: update on early diagnosis and treatment. Am J Psychiatry 1990; 147(6): 696710 .

[21] Breitbart W, Marotta R, Platt M, et al. A double-blind trial of haloperidol, chlorpromazine, and lorazepam in the treatment of delirium in hospitalized AIDS patients. Am J Psychiatry 1996; 153(2): 231-7.

[22] Rogers MP, Liang MH, Daltroy LH, et al. Delirium after elective orthopedic surgery: risk factors and natural history. Int J Psychiatry Med 1989; 19(2): 109-21.

[23] Olofsson B, Stenvall M, Lundstrom M, Gustafson Y, Svensson O. Mental status and surgical methods in patients with femoral neck fracture. Orthop Nurs 2009; 28(6): 305-13.

[24] Gustafson Y. Postoperative delirium--a challenge for the orthopedic team. Acta Orthop Scand 2004; 75(4): 375-7.

[25] Moraga A, Rodriguez-Pascual C. Acurate diagnosis of delirium in elderly patients. Curr Opin Psychiatry 2007; 20(3): 262-7.

[26] Morandi A, Jackson JC, Ely EW. Delirium in the intensive care unit. Int Rev Psychiatry 2009; 21(1): 43-58

[27] Stagno D, Gibson C, Breitbart W. The delirium subtypes: a review of prevalence, phenomenology, pathophysiology, and treatment response. Palliat Support Care 2004; 2(2): 171-9.

[28] Sidhu K, Balon R, Ajluni V, Boutros N. Standard EEG and the difficult-to-assess mental status. Ann Clin Psychiatry 2009; 21(2): 103-8.

[29] Rabins P, Folstein M. Delirium and dementia: diagnostic criteria and fatality rates. Br J Psychiatry1982; 140: 149-53.

[30] Varsamis J, Zuchowski T, Maini KK. Survival rates and causes of death in geriatric psychiatric patients. A six-year follow-up study. Can Psychiatr Assoc J 1972; 17(1): 17-22.

[31] Trzepacz PT, Teague GB, Lipowski ZJ. Delirium and other organic mental disorders in a general hospital. Gen Hosp Psychiatry 1985; 7(2): 101-6.

[32] Cameron DJ, Thomas RI, Mulvihill M, Bronheim H. Delirium: a test of the diagnostic and statistical manual III criteria on medical inpatients. J Am Geriatr Soc 1987; 35(11): 1007-10.

[33] Andrew MK, Freter SH, Rockwood K. Incomplete functional recovery after delirium in elderly people: a prospective cohort study. BMC Geriatr 2005; 17:1-8.

[34] Eeles E, Rockwood K. Delirium in the long-term care setting: clinical and research challenges. J Am Med Dir Assoc 2008; 9(3): 157-61.

[35] McCusker J, Cole M, Abrahamowicz M, Han L, Podoba JE, Ramman-Haddad L. Environmental risk factors for delirium in hospitalized older people. J Am Geriatr Soc 200; 49(10): 1327-34.

[36] Francis J, Kapoor WN. Delirium in hospitalized elderly. J Gen Intern Med 1990; 5(1): 65-79.
[37] Inouye SK. The dilemma of delirium: clinical and research controversies regarding diagnosis and evaluation of delirium in hospitalized elderly medical patients. Am J Med 1994; 97(3): 27888.

[38] Han L, McCusker J, Cole M, Abrahamowicz M, Primeau F, Elie M. Use of medications with anticholinergic effect predicts clinical severity of delirium symptoms in older medical inpatients. Arch Intern Med 2001; 161(8): 1099-105.

[39] Kalisvaart KJ, de Jonghe JF, Bogaards MJ, et al. Haloperidol prophylaxis for elderly hip-surgery patients at risk for delirium: a randomized placebo-controlled study. J Am Geriatr Soc 2005; 53(10): 1658-66.

[40] Jacobi J, Fraser GL, Coursin DB, et al. Clinical practice guidelines for the sustained use of sedatives and analgesics in the critically ill adult. Crit Care Med 2002; 30(1): 119-41.

[41] Han CS, Kim YK. A double-blind trial of risperidone and haloperidol for the treatment of delirium. Psychosomatics 2004; 45(4): 297-301.

[42] Attard A, Ranjith G, Taylor D. Delirium and its treatment. CNS Drugs 2008; 22(8): 631-44.

[43] Skrobik YK, Bergeron N, Dumont M, Gottfried SB. Olanzapine vs haloperidol: treating delirium in a critical care setting. Intens Care Med 2004; 30(3):444-9.

[44] Devlin JW, Roberts RJ, Fong JJ, et al. Efficacy and safety of quetiapine in critically ill patients with delirium: A prospective, multicenter, randomized, double-blind, placebo-controlled pilot study*. Crit Care Med 2009.

[45] Lonergan E, Luxenberg J, Areosa SA. Benzodiazepines for delirium. Cochrane Database Syst Rev 2009; 7(4): CD006379.

[46] Tesar GE. The agitated patient, Part II: Pharmacologic treatment Hosp Commun Psychiatry 1993; 44(7): 627-9

[47] Liptzin B. Delirium. Arch Fam Med 1995; 4(5): 453-8.

[48] Levenson JL. High-dose intravenous haloperidol for agitated delirium following lung transplantation. Psychosomatics. 1995; 36(1): 66-8.

[49] Lawrence KR, Nasraway SA. Conduction disturbances associated with administration of butyrophenone antipsychotics in the critically ill: a review of the literature. Pharmacotherapy 1997; 17(3): 531-7.

[50] Hunt N, Stern TA. The association between intravenous haloperidol and Torsades de Pointes. Three cases and a literature review. Psychosomatics 1995; 36(6): 541-9.

[51] Metzger E, Friedman R. Prolongation of the corrected QT and torsades de pointes cardiac arrhythmia associated with intravenous haloperidol in the medically ill. J Clin Psychopharmacol 1993; 13(2): 128-32.

[52] Lacasse H, Perreault M, Williamson D. Systematic review of antipsychotics for the treatment of hospital-associated delirium in medically or surgically ill patients. Ann Pharmacother 2006; 40(11): 1966-73.

[53] Marcantonio ER, Goldman L, Mangione CM, et al. A clinical prediction rule for delirium after elective noncardiac surgery. JAMA 1994; 271(2): 134-9.

[54] Cancelli I, Beltrame M, Gigli GL, Valente M. Drugs with anticholinergic properties: cognitive and neuropsychiatric sideeffects in elderly patients. Neurol Sci 2009; 30(2): 87-92.

[55] Greene LT. Physostigmine treatment of anticholinergic-drug depression in postoperative patients. Anesth Analg 1971; 50(2): 222-6.

[56] Shapiro BA, Warren J, Egol AB, et al. Practice parameters for intravenous analgesia and sedation for adult patients in the intensive care unit: an executive summary. Society of Critical Care Medicine. Crit Care Med 1995; 23(9): 1596-600.

[57] Tune L, Carr S, Cooper T, Klug B, Golinger RC. Association of anticholinergic activity of prescribed medications with postoperative delirium. J Neuropsychiatry Clin Neurosci 1993; 5(2): 208-10

[58] Tune L, Carr S, Hoag E, Cooper T. Anticholinergic effects of drugs commonly prescribed for the elderly: potential means for assessing risk of delirium. Am J Psychiatry 1992; 149(10): 1393-4.

[59] Someya T, Shibasaki M, Noguchi T, Takahashi S, Inaba $T$. Haloperidol metabolism in psychiatric patients: importance of glucuronidation and carbonyl reduction. J Clin Psychopharmacol 1992;12(3): 169-74. 
[60] Hriso E, Kuhn T, Masdeu JC, Grundman M. Extrapyramidal symptoms due to dopamine-blocking agents in patients with AIDS encephalopathy. Am J Psychiatry 1991;148(11): 1558-61.

[61] Meagher DJ, O'Hanlon D, O'Mahony E, Casey PR. The use of environmental strategies and psychotropic medication in the management of delirium. Br J Psychiatry 1996; 168(4): 512-5.

[62] Cole MG, McCusker J, Bellavance F, et al. Systematic detection and multidisciplinary care of delirium in older medical inpatients: a randomized trial. CMAJ 2002; 167(7): 753-9.
[63] Budd S, Brown W. Effect of a reorientation technique on postcardiotomy delirium. Nurs Res 1974; 23(4): 341-8.

[64] Inouye SK, Bogardus ST Jr, Charpentier PA, et al. A multicomponent intervention to prevent delirium in hospitalized older patients. N Engl J Med 1999; 340(9): 669-76.

[65] Inouye SK, VanDyck $\mathrm{CH}$, Alessi CA, et al. Clarifying confusion: The Confusion Assessment Method. A new method for detecting delirium. Ann Intern Med 1990; 113: 941-8.

Received: January 18,2010

Revised: March 16, 2011

Accepted: April 15, 2011

(C) Jochum and Bär; Licensee Bentham Open.

This is an open access article licensed under the terms of the Creative Commons Attribution Non-Commercial License (http://creativecommons.org/licenses/ by-nc/3.0/) which permits unrestricted, non-commercial use, distribution and reproduction in any medium, provided the work is properly cited. 